\section{New and improved dystrophic mice}

\section{By Tim Fulmer, Senior Writer}

California researchers have developed a new mouse model of Duchenne muscular dystrophy that better reflects the disease than current rodent models. ${ }^{1}$ The team plans to use the model to identify combination therapies that not only correct the genetic defect underlying the disease but also block its progression.

The standard model of DMD is the $m d x$ mouse, which carries the mutated dystrophin (Dmd; $m d x$ ) gene found in patients. However, there is a well-known discrepancy between the severe muscle weakness of DMD patients and the relatively mild symptoms of the $m d x$ mouse.

A team led by Helen Blau and Jason Pomerantz hypothesized that differences in mouse and human telomeres might underlie the discrepancy. Telomeres are protein-DNA structures that cap the ends of chromosomes to protect them from damage and prevent instability of the genome. Excessive shortening of telomeres can lead to cellular senescence., ${ }^{2,3}$

Previous studies have shown that muscle cells from DMD patients have less potential to proliferate and regenerate, ${ }^{4,5}$ which in some cases is associated with shortening of telomeres, than muscle cells from healthy subjects. ${ }^{6,7}$

The researchers decided to test their hypothesis by creating an $m d x$ mouse with shortened telomeres. The resulting double knockout mice were deficient in $m d x$ and telomerase RNA component (Terc; $m T R$ ), a portion of the telomerase enzyme that ensures chromosomes maintain proper telomere lengths.

Compared with single knockout and wild-type mice, the double knockouts showed increased disease severity based on several different criteria.

For example, the $m d x / m T R$ mice had significantly higher levels of serum creatine kinase than control mice that lacked only $m d x$ or only $m T R$ $(p<0.05$ and $p<0.0001$, respectively). Increased creatine kinase is a marker of skeletal muscle damage in mice and is used to diagnose DMD.

The $m d x / m T R$ mice also became exhausted more quickly on a treadmill than controls ( $p<0.0001$ for both single knockout controls) and had more damage to their diaphragm and calf muscles, which showed extensive fibrosis, calcium deposits and immune cell infiltration. Finally, disease in the $m d x / m T R$ mice progressed more rapidly with age and ultimately led to a shortened lifespan.
Further cell culture and in vivo studies showed that the severe disease phenotype in the double knockouts was associated with a decreased capacity of muscle stem cells to proliferate.

The authors wrote that the data "....indicate that the combination of the structural defect of dystrophin deficiency that leads to muscle degeneration together with the progressive exhaustion of functional MuSC [muscle stem cells] generates the dystrophic phenotype."

The findings were published in Cell.

"The findings from the $m d x / m T R$ mice change the way we view treating Duchenne muscular dystrophy. We can no longer focus solely on correcting the dystrophin deficiency," Blau told SciBX. "Presumably the ideal therapeutic strategy should address a twofold deficiency-the lack of a functional dystrophin gene and the lack of muscle stem cells capable of proliferating and replenishing muscle tissue."

Blau is director of the Baxter Laboratory for Stem Cell Biology and a professor of microbiology and immunology at the Stanford University School of Medicine. Pomerantz is an assistant professor in the Division of Plastic and Reconstructive Surgery at the University of California, San Francisco.

In a commentary accompanying the paper, Jeffrey Chamberlain wrote, "The $m d x / m T R$ double-mutant mice increase the power of therapeutic testing by providing greater resolution between normal and dystrophic muscle function." ${ }^{8}$ Chamberlain is a professor of neurology, medicine and biochemistry at the University of Washington School of Medicine.

\section{One-two punch for DMD}

Blau and colleagues now want to use the mice as disease models to identify therapeutic strategies that target both aspects of DMD-loss of muscle cell regenerative capacity and dystrophin deficiency.

"One can already imagine the general outlines of such a dual therapy-gene therapy to correct the dystrophin deficiency and stem cell therapy to correct the loss of muscle stem cells," Blau said.

Blau and Chamberlain have set up a collaboration to use the $m d x / m T R$ mice to identify such dual therapies. The partnership combines Blau and Pomerantz's focus on muscle stem cells with Chamberlain's focus on gene therapy to treat muscular dystrophy.

In addition, the researchers want to use the mice to identify markers associated with muscle stem cell loss in disease and aging. "Based on the Cell paper, we already have one such marker-shortened telomere length," Pomerantz told SciBX. "However, we gather from prior studies on muscle stem cell aging that there are multiple markers of loss of stem cell proliferative capacity, including elevated oxidative damage and increased levels of the tumor suppressor p16."

Ideally, a panel of such biomarkers would allow staging of DMD progression, said Pomerantz. "Using the markers to help us differentiate early stage from late stage disease will probably be important to help guide the choice of the right DMD therapy," he said. 


\section{ANALYSIS/PROSPECTS}

Chamberlain agreed. In very young children with early stage disease, when the muscle stem cells have not yet become exhausted, gene therapy to correct the dystrophin deficiency might be sufficient, he said. In older children, in which disease has progressed and significant muscle stem cell exhaustion has occurred, dual gene therapy and cell therapy will likely be necessary, he said.

Finally, Blau said, a third area of interest is using the $m d x / m T R$ mouse model to screen small molecule and peptide libraries for compounds that trigger endogenous muscle stem cells to proliferate.

"Such compounds could be valuable not only for preventing muscle stem cell exhaustion associated with muscular dystrophy but could also be useful for reversing muscle stem cell loss associated with injury and aging," she said.

Blau said the findings published in Cell are covered by patents but declined to disclose further details.

Fulmer, T. SciBX 4(3); doi:10.1038/scibx.2011.65

Published online Jan. 20, 2011

\section{REFERENCES}

1. Sacco, A. et al. Cell; published online Dec. 9, 2010; doi:10.1016/j.cell.2010.11.039

Contact: Helen M. Blau, Stanford University School of Medicine, Stanford, Calif.

e-mail: hblau2@stanford.edu

Contact: Jason H. Pomerantz, University of California,

San Francisco, Calif.

e-mail: jason.pomerantz@ucsfmedctr.org

2. Sherr, C.J. \& DePinho, R.A. Cell 102, 407-410 (2000)

3. Rodier, F. et al. Int. J. Biochem. Cell Biol. 37, 977-990 (2005)

4. Blau, H.M. et al. Proc. Natl. Acad. Sci. USA 80, 4856-4860 (1983)

5. Webster, C. \& Blau, H.M. Somat. Cell Mol. Genet. 16, 557-565 (1990)

6. Decary, S. et al. Neuromuscul. Disord. 10, 113-120 (2000)

7. Mouly, V. et al. Acta Physiol. Scand. 184, 3-15 (2005)

8. Chamberlain, J.S. Cell 143, 1040-1042 (2010)

\section{COMPANIES AND INSTITUTIONS MENTIONED}

Stanford University School of Medicine, Stanford, Calif. University of California, San Francisco, Calif.

University of Washington School of Medicine, Seattle, Wash. 